\title{
Analytic partial orders and oriented graphs
}

\author{
by
}

Alain Louveau (Paris)

\begin{abstract}
We prove that there is no maximum element, under Borel reducibility, in the class of analytic partial orders and in the class of analytic oriented graphs. We also provide a natural jump operator for these two classes.
\end{abstract}

This paper is part of the general program of studying $\boldsymbol{\Sigma}_{1}^{1}$ (analytic) binary relations on Polish spaces, under the Borel reducibility ordering, and variants of it.

If $R_{1}$ and $R_{2}$ are binary relations on Polish spaces $X_{1}$ and $X_{2}$ respectively, a reduction of $R_{1}$ to $R_{2}$ is a map $f: X_{1} \rightarrow X_{2}$ such that for all $x, y$ in $X_{1}, x R_{1} y \leftrightarrow f(x) R_{2} f(y)$.

We say that $R_{1}$ is Borel reducible to $R_{2}$, or $R_{2}$ Borel reduces $R_{1}$, and write $R_{1} \leq_{\mathrm{B}} R_{2}$, if there is a Borel reduction of $R_{1}$ to $R_{2}$. If there is an injective Borel reduction, we say that $R_{1}$ Borel embeds into $R_{2}$ and write $R_{1} \sqsubseteq_{\mathrm{B}} R_{2}$.

If $\mathcal{C}$ is a class of binary relations on Polish spaces, a relation $R$ is $\mathcal{C}$-complete if $R \in \mathcal{C}$ and $R$ Borel reduces all elements of $\mathcal{C}$.

It is known that many natural classes of $\boldsymbol{\Sigma}_{1}^{1}$ binary relations admit complete elements, e.g. the class of $\boldsymbol{\Sigma}_{1}^{1}$ equivalence relations, the class of $\boldsymbol{\Sigma}_{1}^{1}$ quasi-orderings, or the class of $\boldsymbol{\Sigma}_{1}^{1}$ graphs (see [LR]).

There are also known examples of classes with no complete elements. H. Friedman proved that this is the case for Borel equivalence relations (see [FS] and [L1]), and it implies easily that this is also the case for Borel quasi-orderings. Another example is the class of $G_{\delta}$ quasi-orders, as shown in Louveau [L2].

In this paper, we will add two more examples of this phenomenon to the previous list, maybe somewhat more surprising as they are classes of $\boldsymbol{\Sigma}_{1}^{1}$ objects.

2000 Mathematics Subject Classification: Primary 03E15, 04A15.

Key words and phrases: analytic partial orders, analytic graphs, Borel reducibility. 
First, we will consider the case of $\boldsymbol{\Sigma}_{1}^{1}$ partial orders, i.e. transitive, reflexive relations $R$ such that $x R y$ and $y R x$ imply $x=y$. We will prove:

Theorem 1. The class $\mathcal{C}$ of $\boldsymbol{\Sigma}_{1}^{1}$ partial orders admits no complete element. In fact, the Borel partial orders are unbounded in $\mathcal{C}$ for the ordering $\leq_{\mathrm{B}}$.

This result will be obtained by considering more general binary relations.

If $R$ is a partial order, its strict part $<_{R}$ is defined by $x<_{R} y \leftrightarrow$ $x R y$ and $x \neq y$. It is a strict order, i.e. a transitive irreflexive relation. And conversely, for each strict order $S$, the relation $x S y$ or $x=y$ is a partial order admitting $S$ as its strict part. Moreover, if $f$ is a reduction of the partial order $R_{1}$ to the partial order $R_{2}$, then $f$ must reduce equality to equality, i.e. be injective, and also reduce $<_{R_{1}}$ to $<_{R_{2}}$. And as the converse also holds, we easily see that

$$
R_{1} \leq_{\mathrm{B}} R_{2} \leftrightarrow<_{R_{1}} \sqsubseteq_{\mathrm{B}}<_{R_{2}} .
$$

Strict orders are a particular case of oriented graphs, those binary relations $R$ which are antisymmetric, i.e. satisfy $x R y \rightarrow \neg y R x$. And for these relations, there is another interesting natural ordering, weaker than Borel reducibility, given by using homomorphisms instead of reductions.

Definition 2. If $R_{1}, R_{2}$ are binary relations on Polish spaces $X_{1}, X_{2}$ respectively, a map $f: X_{1} \rightarrow X_{2}$ is a homomorphism from $R_{1}$ to $R_{2}$ if for all $x, y$ in $X_{1}$,

$$
x R_{1} y \rightarrow f(x) R_{2} f(y) .
$$

We write $R_{1} \preceq_{\mathrm{B}} R_{2}$ if there is a Borel homomorphism from $R_{1}$ to $R_{2}$.

From the above discussion, Theorem 1 is an immediate consequence of the following

TheOREM 3. No $\boldsymbol{\Sigma}_{1}^{1}$ oriented graph can bound all Borel strict orders in $\preceq_{\mathrm{B}}$. In particular, the class of $\boldsymbol{\Sigma}_{1}^{1}$ oriented graphs and the class of $\boldsymbol{\Sigma}_{1}^{1}$ strict orders admit no complete element (for $\preceq_{\mathrm{B}}$, hence also for $\leq_{\mathrm{B}}$ ).

In order to prove Theorem 3, we use an "index method". We first define for each $\boldsymbol{\Sigma}_{1}^{1}$ oriented graph $R$ a countable ordinal ind $(R)$ satisfying

$$
R_{1} \preceq_{\mathrm{B}} R_{2} \rightarrow \operatorname{ind}\left(R_{1}\right) \leq \operatorname{ind}\left(R_{2}\right) .
$$

Then we prove that this index is unbounded on the class of Borel strict orders. These facts together of course prove Theorem 3.

To define the index, recall from [L1] the notion of potential Borel class: A binary relation $R$ on a Polish space $X$ is potentially $\boldsymbol{\Delta}_{\xi}^{0}$ if there is a finer Polish topology $\tau$ on $X$ such that $R$ is $\Delta_{\xi}^{0}$ in the square of $(X, \tau)$. Note that if $f: X_{1} \rightarrow X_{2}$ is a Borel map and $R$ is potentially $\boldsymbol{\Delta}_{\xi}^{0}$ on $X_{2}$, so is $f^{-1}(R)$ on $X_{1}$. For one can first refine the topology of $X_{2}$ so that $R$ is $\boldsymbol{\Delta}_{\xi}^{0}$, and then 
the topology of $X_{1}$ to make $f$ continuous with respect to the new Polish topology of $X_{2}$.

This leads to the following definition, where $\check{R}$ denotes the dual of $R$, defined by $x \check{R} y$ whenever $y R x$.

Definition 4. If $R$ is a $\Sigma_{1}^{1}$ oriented graph, let ind $(R)$ be the least ordinal $\xi<\omega_{1}$ such that there exists a potentially $\boldsymbol{\Delta}_{\xi}^{0}$ set $C$ separating $R$ from $\check{R}$, i.e. satisfying $R \subseteq C$ and $C \cap \check{R}=\emptyset$.

Note that $\operatorname{ind}(R)$ is well defined for each $\boldsymbol{\Sigma}_{1}^{1}$ oriented graph $R$, because $R$ and $\check{R}$ are two disjoint $\boldsymbol{\Sigma}_{1}^{1}$ sets, hence Borel separable. And the above remarks imply immediately that the index ind is increasing, i.e. $R_{1} \preceq_{\mathrm{B}} R_{2}$ implies ind $\left(R_{1}\right) \leq \operatorname{ind}\left(R_{2}\right)$, for a Borel homomorphism $f$ from $R_{1}$ to $R_{2}$ is also a homomorphism from $\check{R}_{1}$ to $\check{R}_{2}$ and hence if $C$ is a potentially $\boldsymbol{\Delta}_{\xi}^{0}$ set separating $R_{2}$ from $\check{R}_{2}$, then $f^{-1}(C)$ is a potentially $\boldsymbol{\Delta}_{\xi}^{0}$ set which separates $R_{1}$ from $\check{R}_{1}$.

So to get Theorem 3 , it is enough to build a family $\left(R_{\xi}\right)$ of Borel strict orders with $\operatorname{ind}\left(R_{\xi}\right)>\xi$, at least for $\xi$ a successor ordinal $\geq 2$, as we now proceed to do. First we define the domain $X_{\xi}$ of $R_{\xi}$ as $2^{D_{\xi} \times \omega}$, where $D_{\xi}$ is a countable set, defined inductively by

$$
\begin{aligned}
& D_{2}=\{0\}, \\
& D_{\xi+1}= \begin{cases}\omega \times D_{\xi} & \text { if } \xi \geq 2 \text { is successor, } \\
\left\{(n, i): i \in D_{\xi_{n}}\right\} & \text { if } \xi \text { is limit and } \xi_{n} \text { is a sequence of } \\
& \text { successors converging to } \xi .\end{cases}
\end{aligned}
$$

Note that the two cases in this definition are the same if we set $\xi_{n}=\xi$ for all $n$, when $\xi$ is successor. And in both cases we can (and will) view each $\alpha \in X_{\xi+1}$ as a sequence $\left(\alpha_{n}\right)_{n \in \omega}$, with $\alpha_{n} \in X_{\xi_{n}}$ for all $n$. With these conventions, we are now in a position to define inductively the strict orders $R_{\xi}$, together with Borel sets $L_{\xi}$ and $L_{\xi}^{*}$, as follows:

CASE $\xi=2$. Recall that the equivalence relation $E_{0}$ is defined on $2^{\omega}$ by

$$
\alpha E_{0} \beta \leftrightarrow \forall^{*} n \alpha(n)=\beta(n),
$$

where $\forall^{*}$ is the quantifier "for all but finitely many".

We have $X_{2}=2^{\omega}$, and set

$$
\begin{array}{r}
R_{2}=\left\{(\alpha, \beta): \alpha \neq \beta \text { and } \alpha E_{0} \beta \text { and if } n\right. \text { is maximum } \\
\text { with } \alpha(n) \neq \beta(n), \text { then } \alpha(n)<\beta(n)\}, \\
L_{2}=\left\{\alpha: \alpha E_{0} \underline{0}\right\}, \quad L_{2}^{*}=\left\{\alpha: \alpha E_{0} \underline{1}\right\},
\end{array}
$$

where $\underline{0}$ and $\underline{1}$ are the reals which are identically 0 and 1 respectively.

The order $R_{2}$ is the strict part of the partial order called $\leq_{0}$ in Kanovei $[\mathrm{K}]$. It orders all $E_{0}$-classes (except $L_{2}$ and $L_{2}^{*}$ ) in order type $\mathbb{Z}$. 
INDUCTIVE CASE. Using the conventions above, for $\xi \geq 2$ we set

$$
\begin{aligned}
& R_{\xi+1}=\left\{(\alpha, \beta) \in X_{\xi+1}^{2}: \forall^{*} n \alpha_{n} R_{\xi_{n}} \beta_{n}\right\}, \\
& L_{\xi+1}=\left\{\alpha \in X_{\xi+1}: \forall n\left(\alpha_{n} \in L_{\xi_{n}} \text { or } \alpha_{n} \in L_{\xi_{n}}^{*}\right) \text { and } \forall^{*} n\left(\alpha_{n} \in L_{\xi_{n}}\right)\right\}, \\
& L_{\xi+1}^{*}=\left\{\alpha \in X_{\xi+1}: \forall n\left(\alpha_{n} \in L_{\xi_{n}} \text { or } \alpha_{n} \in L_{\xi_{n}}^{*}\right) \text { and } \forall^{*} n\left(\alpha_{n} \in L_{\xi_{n}}^{*}\right)\right\} .
\end{aligned}
$$

One easily checks that each $R_{\xi}$ is a Borel strict order. So to get Theorem 3 , it is enough to prove that for all successor $\xi \geq 2$, ind $\left(R_{\xi}\right)>\xi$, i.e. that no potentially $\boldsymbol{\Delta}_{\xi}^{0}$ set can separate $R_{\xi}$ from $\check{R}_{\xi}$. We will prove this in two steps, by first proving that there is no separation by a $\boldsymbol{\Delta}_{\xi}^{0}$ set, and then dealing with the possible change of topologies.

For the first step, we use the following lemma, which also explains the notation for the sets $L_{\xi}$ and $L_{\xi}^{*}$ : the letter $L$ is for Lebesgue (the same idea was already used in [HKL] for other purposes).

Lemma 5. Let $\xi>1$ be successor. There is no $\boldsymbol{\Delta}_{\xi}^{0}$ set separating $L_{\xi}$ from $L_{\xi}^{*}$.

Proof. This is a direct consequence of Lebesgue's classical result about the generation of Baire class $\xi$ functions by the operation of taking pointwise limits: For $\xi \geq 1$, Baire class $\xi$ functions are the pointwise limits of sequences of functions of Baire class $<\xi$, and even of Baire class $<\lambda$ if $\xi=\lambda+1$ with $\lambda$ limit. This result is valid for real-valued functions on arbitrary Polish spaces, but it is also valid for $\{0,1\}$-valued functions on $\operatorname{dim} 0$ Polish spaces, in particular for Borel subsets of $2^{\omega}$. And there, tracing back in the inductive definition above, one easily checks that it exactly means that for any successor $\xi \geq 2$ and any $\boldsymbol{\Delta}_{\xi}^{0}$ set $C \subseteq 2^{\omega}$, there is a sequence $\left(C_{i, n}\right)_{(i, n) \in D_{\xi} \times \omega}$ of clopen subsets of $2^{\omega}$ such that

(i) for each $\alpha \in 2^{\omega}, 1_{\left\{(i, n): \alpha \in C_{i, n}\right\}} \in L_{\xi} \cup L_{\xi}^{*}$,

(ii) $\alpha \in C \leftrightarrow 1_{\left\{(i, n): \alpha \in C_{i, n}\right\}} \in L_{\xi}^{*}$

But then, as the map $\alpha \mapsto 1_{\left\{(i, n): \alpha \in C_{i, n}\right\}}$ is continuous, we find that if some $\boldsymbol{\Delta}_{\xi}^{0}$ set $C$ were separating $L_{\xi}^{*}$ from $L_{\xi}$, every $\boldsymbol{\Delta}_{\xi}^{0}$ subset of $2^{\omega}$ would be in the Wadge class of $C$, which, as $\xi \geq 2$, is a contradiction proving the lemma.

Using this lemma, we get

Proposition 6. For each successor ordinal $\xi \geq 2$, there is a continuous map $f_{\xi}=\left(f_{\xi}^{0}, f_{\xi}^{1}\right): X_{\xi} \rightarrow X_{\xi} \times X_{\xi}$ satisfying:

(i) if $\alpha \in L_{\xi}$, then $f_{\xi}^{0}(\alpha) R_{\xi} f_{\xi}^{1}(\alpha)$,

(ii) if $\alpha \in L_{\xi}^{*}$, then $f_{\xi}^{1}(\alpha) R_{\xi} f_{\xi}^{0}(\alpha)$.

In particular, $R_{\xi}$ cannot be separated from $\check{R}_{\xi}$ by a $\Delta_{\xi}^{0}$ set. 
Proof. The second assertion follows from the first and Lemma 5: any separating $\boldsymbol{\Delta}_{\xi}^{0}$ set would yield, by taking the inverse image under $f_{\xi}$, a $\boldsymbol{\Delta}_{\xi}^{0}$ set separating $L_{\xi}$ from $L_{\xi}^{*}$, contradicting Lemma 5 .

To prove the first assertion, we define $f_{\xi}$ by induction.

For $\xi=2, \beta^{0}=f_{2}^{0}(\alpha)$ and $\beta^{1}=f_{2}^{1}(\alpha)$ are defined by:

(i) for $n=0$,

$$
\beta^{0}(0)=\alpha(0), \quad \beta^{1}(0)=1-\alpha(0)
$$

(ii) for $n>0$,

(0) if $\alpha(n)=\alpha(n-1)$, then $\beta^{0}(n)=\beta^{1}(n)=0$,

(1) if $\alpha(n)<\alpha(n-1)$, then $\beta^{0}(n)=0$ and $\beta^{1}(n)=1$,

(2) if $\alpha(n)>\alpha(n-1)$, then $\beta^{0}(n)=1$ and $\beta^{1}(n)=0$.

To check it works, suppose first $\alpha \in L_{2}$, and let $n$ be smallest with $\alpha(k)=0$ for $k \geq n$. Then, whether $n=0$ or not, we are in case (1) of the definition at $n$, and in case $(0)$ at all $k>n$. This implies that $n$ is largest with $\beta^{0}(n) \neq \beta^{1}(n)$. As $\beta^{0}(n)<\beta^{1}(n)$, we get $\beta^{0}(n) R_{2} \beta^{1}(n)$ as wanted.

Suppose now $\alpha \in L_{2}^{*}$, and let $n$ be smallest with $\alpha(k)=1$ for $k \geq n$. Then we are in case (2) of the definition at $n$, and in case (0) at any $k>n$. Again $n$ is largest with $\beta^{0}(n) \neq \beta^{1}(n)$, and as $\beta^{0}(n)>\beta^{1}(n)$, we get $\beta^{1}(n) R_{2} \beta^{0}(n)$ as wanted.

This gives the proposition for $\xi=2$.

The induction step is easy. Using the same conventions as before, for $\alpha=\left(\alpha_{n}\right)_{n \in \omega}$ in $X_{\xi}$, set $f_{\xi}^{i}(\alpha)=\left(f_{\xi_{n}}^{i}\left(\alpha_{n}\right)\right)_{n \in \omega}$ for $i=0,1$.

By induction we find that if $\alpha \in L_{\xi}$, then for all but finitely many $n$ 's, $\alpha_{n} \in L_{\xi_{n}}$, hence for the same $n$ 's, $f_{\xi_{n}}^{0}\left(\alpha_{n}\right) R_{\xi_{n}} f_{\xi_{n}}^{1}\left(\alpha_{n}\right)$, so $f_{\xi}^{0}(\alpha) R_{\xi} f_{\xi}^{1}(\alpha)$, and similarly with $L_{\xi}^{*}$ and $\check{R}_{\xi}$. This proves Proposition 6 .

We now get rid of the possible change of topologies. Fix the ordinal $\xi$. A good pair $(D, \gamma)$ at level $\xi$ consists of a subset $D \subseteq D_{\xi} \times \omega$ such that for all $i \in D_{\xi}$, the set $D_{i}=\{n:(i, n) \in D\}$ is infinite, together with a map $\gamma:\left(D_{\xi} \times \omega\right)-D \rightarrow 2$.

Such a pair $(D, \gamma)$ defines a compact set

$$
K_{D, \gamma}=\left\{\alpha \in X_{\xi}:\left.\alpha\right|_{\left(D_{\xi} \times \omega\right)-D}=\gamma\right\},
$$

and a natural homeomorphism $h_{D, \gamma}=h$ of $X_{\xi}$ onto $K_{D, \gamma}$ : if for $i \in D_{\xi}, d_{i}$ is the increasing enumeration of $D_{i}$, define $\beta=h(\alpha)$ by $\beta(i, n)=\alpha\left(i, d_{i}^{-1}(n)\right)$ if $(i, n) \in D$ and $\beta(i, n)=\gamma(i, n)$ otherwise.

Lemma 7. For each successor ordinal $\xi>1$ and $\operatorname{good} \operatorname{pair}(D, \gamma)$ at level $\xi$, the map $h_{D, \gamma}$ is a continuous reduction of $R_{\xi}$ to $\left.R_{\xi}\right|_{K_{D, \gamma}}$.

Proof. We argue by induction. If $\xi=2$, then $D$ is an infinite subset of $\omega$ with enumeration $d$, and given $\alpha$ and $\beta$ in $X_{2}=2^{\omega}$, the set of integers where $h(\alpha)$ and $h(\beta)$ differ is the $d$-image of the set of integers where $\alpha$ and 
$\beta$ differ. So we get $\alpha \neq \beta$ if and only if $h(\alpha) \neq h(\beta), \alpha E_{0} \beta$ if and only if $h(\alpha) E_{0} h(\beta)$, and, as $d$ is increasing, the largest $n$ where $h(\alpha)$ and $h(\beta)$ differ, when it exists, is the image under $d$ of the largest $n$ where $\alpha$ and $\beta$ differ. So finally $\alpha R_{2} \beta$ if and only if $h(\alpha) R_{2} h(\beta)$.

Again the induction step is easy. Fix $(D, \gamma)$ good at level $\xi$. Recall that with our conventions, $D_{\xi}=\left\{(n, i): i \in D_{\xi_{n}}\right\}$. So for each $n$, we get a pair at level $\xi_{n}$ by setting $D_{n}=\left\{(i, k) \in D_{\xi_{n}} \times \omega:((n, i), k) \in D\right\}$ and $\gamma_{n}((i, k))=\gamma((n, i), k)$ for $(i, k) \in D_{n}$. Clearly $\left(D_{n}, \gamma_{n}\right)$ is good at level $\xi_{n}$, so by induction the corresponding homeomorphism $h_{n}$ is such that for $\alpha, \beta$ in $X_{\xi_{n}}, \alpha R_{\xi_{n}} \beta$ if and only if $h_{n}(\alpha) R_{\xi_{n}} h_{n}(\beta)$.

But note that for $\alpha=\left(\alpha_{n}\right)_{n \in \omega}$ in $X_{\xi}$, one has $h(\alpha)=\left(h_{n}\left(\alpha_{n}\right)\right)_{n \in \omega}$, so that the previous fact implies immediately that $\alpha R_{\xi} \beta$ if and only if $h(\alpha) R_{\xi} h(\beta)$, as desired.

To finish the proof of Theorem 3, we need the following (essentially classical) lemma:

Lemma 8. Let $C$ be a countable set, and $H$ a dense $G_{\delta}$ subset of $2^{C \times \omega}$. Then there exists a subset $D$ of $C \times \omega$ with $D_{i}$ infinite for all $i \in C$, and $a$ map $\gamma:(C \times \omega)-D \rightarrow 2$ such that

$$
K_{D, \gamma}=\left\{\alpha \in 2^{C \times \omega}:\left.\alpha\right|_{(C \times \omega)-D}=\gamma\right\}
$$

is a subset of $H$.

Proof. The pair $(D, \gamma)$ is constructed by induction. Say that $(d, g)$ is a finite approximation if $d$ and $\operatorname{dom}(g)$ are finite disjoint subsets of $C \times \omega$. It is clearly enough to check that given a finite approximation $(d, g)$, an $i \in C$ and a dense open set $U \subseteq 2^{C \times \omega}$, one can extend $(d, g)$ to some $\left(d^{\prime}, g^{\prime}\right)$ so that $d^{\prime}-d$ meets $\{i\} \times \omega$, and the clopen set $V_{g^{\prime}}=\left\{\alpha \in 2^{C \times \omega}:\left.\alpha\right|_{\operatorname{dom}\left(g^{\prime}\right)}=g^{\prime}\right\}$ is a subset of $U$. But this is easy: Pick first $n$ with $(i, n)$ outside $d \cup \operatorname{dom}(g)$, and set $d^{\prime}=d \cup\{(i, n)\}$. Enumerate $2^{d^{\prime}}$ as $f_{1}, \ldots, f_{N}$, and define inductively $g_{0}, g_{1}, \ldots, g_{N}$ so that they all have their domains disjoint from $d^{\prime}, g_{0}=g$ and they extend each other, and for each $k \leq N, V_{f_{k} \cup g_{k}} \subseteq U$. This is possible by the density of $U$. But then $g^{\prime}=g_{N}$ works.

End of proof of Theorem 3. As said before, we just have to check that for any successor ordinal $\xi \geq 2$, $\operatorname{ind}\left(R_{\xi}\right)>\xi$. Argue by contradiction, and suppose $C$ is a Borel set separating $R_{\xi}$ from $\check{R}_{\xi}$, and $\tau$ a finer Polish topology on $X_{\xi}$ such that $C$ is $\boldsymbol{\Delta}_{\xi}^{0}$ in $\left(X_{\xi}, \tau\right)^{2}$. Fix then a dense $G_{\delta}$ subset $H$ of $X_{\xi}$ on which $\tau$ and the usual topology coincide. Applying Lemma 8 (with $D_{\xi}$ ), we get a good pair $(D, \gamma)$ at level $\xi$ with $K=K_{D, \gamma} \subseteq H$. But then $C \cap K^{2}$ is $\boldsymbol{\Delta}_{\xi}^{0}$, and by Lemma $7, h_{D, \gamma}^{-1}(C)$ is a $\boldsymbol{\Delta}_{\xi}^{0}$ set separating $R_{\xi}$ from $\check{R}_{\xi}$, contradicting Proposition 6. 
REMARK. The minimal expected complexity of a strict order $R$ with $\operatorname{ind}(R)>\xi$ is $\boldsymbol{\Sigma}_{\xi}^{0}\left(\boldsymbol{\Pi}_{\xi}^{0}\right.$ is not possible because of the separation property of that class). Our examples are more complicated, except for $\xi=2$ and $\xi$ successor of a limit ordinal. We do not know if $\boldsymbol{\Sigma}_{\xi}^{0}$ is always obtainable.

It is often the case with the index method (see [L1]) that the actual proof of the unboundedness of the index provides a jump operator. This is also the case here, at least in spirit, for we will need a slight variant of the previous proof to get the strongest possible jump result.

If $\left(R_{n}\right)_{n \in \omega}$ is a sequence of $\boldsymbol{\Sigma}_{1}^{1}$ oriented graphs on Polish spaces $X_{n}$, define a $\boldsymbol{\Sigma}_{1}^{1}$ oriented graph $\left(R_{n}\right)^{+}$on $\prod_{n} X_{n}$ by

$$
x\left(R_{n}\right)^{+} y \leftrightarrow \forall^{*} n x_{n} R_{n} y_{n} .
$$

The operator + is clearly increasing for the orderings $\preceq_{\mathrm{B}}$ and $\leq_{\mathrm{B}}$ : if for all $n, R_{n} \preceq_{\mathrm{B}} S_{n}$ (resp. $R_{n} \leq_{\mathrm{B}} S_{n}$ ), one also has $\left(R_{n}\right)^{+} \preceq_{\mathrm{B}}\left(S_{n}\right)^{+}$(resp. $\left.\left(R_{n}\right)^{+} \leq_{\mathrm{B}}\left(S_{n}\right)^{+}\right)$, by combining the witnessing maps.

If for all $n, R_{n}=R$, write $R^{+}$instead of $\left(R_{n}\right)^{+}$. This defines a $\preceq$ - and $\leq_{\mathrm{B}}$-increasing operator, which clearly satisfies $R \leq_{\mathrm{B}} R^{+}$for all $\Sigma_{1}^{1}$ oriented graphs $R$.

The next result is a direct consequence of the proof of the unboundedness of the index. It shows that for complicated enough $\boldsymbol{\Sigma}_{1}^{1}$ oriented graphs, + is in fact a jump operator.

Corollary 9. Let $R$ be a $\boldsymbol{\Sigma}_{1}^{1}$ oriented graph with $R_{2} \preceq_{\mathrm{B}} R$. Then $R^{+} \npreceq_{\mathrm{B}} R$.

Proof. Assume not, and let $R$ be a counterexample. Then from $R^{+} \preceq_{\mathrm{B}} R$ and the fact that + is $\preceq_{\mathrm{B}}$-increasing, one sees easily by induction that for all countable successor ordinals $\xi \geq 2, R_{\xi} \preceq_{\mathrm{B}} R$, contradicting (the proof of) Theorem 3.

The condition $R_{2} \preceq_{\mathrm{B}} R$ in the previous corollary is not optimal, and may look a bit unnatural. We now show that at least in the context of $\boldsymbol{\Sigma}_{1}^{1}$ strict orders, it provides the optimal result. Then we will see how to change the arguments to get the optimal result for arbitrary $\boldsymbol{\Sigma}_{1}^{1}$ oriented graphs.

First, let us consider the particular case of $\boldsymbol{\Sigma}_{1}^{1}$ strict orders. Recall from Kanovei $[\mathrm{K}]$ that a partial order $R$ is Borel linearizable if it admits an extension which is a Borel linear order. We will use the following result from [L3]:

THEOREM 10. The following are equivalent, for a $\boldsymbol{\Sigma}_{1}^{1}$ partial order $R$ :

(i) $R$ is not Borel linearizable,

(ii) $R_{2} \preceq_{\mathrm{B}}<_{R}$

(iii) $\operatorname{ind}\left(<_{R}\right)>2$. 
COROLlary 11. Let $R$ be a $\boldsymbol{\Sigma}_{1}^{1}$ strict order which admits finite chains of arbitrary cardinality. Then $R^{+} \npreceq_{\mathrm{B}} R$.

Proof. By Corollary 9, it is enough to check that any possible counterexample $R$ satisfies $R_{2} \preceq_{\mathrm{B}} R$. By the assumption on $R$, we have for each $n \in \omega$ an increasing $R$-chain $\left(x_{n}^{i}\right)_{i \leq n}$. We then use the following

FACT. One can build in $\omega^{\omega}$ an $\omega_{1}$-sequence $\left(f_{\xi}\right)_{\xi<\omega_{1}}$ such that for all $n$, $f_{\xi}(n) \leq n$, and $\eta<\xi$ implies $\forall^{*} n f_{\eta}(n)<f_{\xi}(n)$.

Granted this fact, the sequence $\left(\left(x_{n}^{f_{\xi}(n)}\right)_{n \in \omega}\right)_{\xi<\omega_{1}}$ is then an increasing $R^{+}$-chain. But it is a result of Harrington, Marker and Shelah [HMS] that in any Borel linear order there is no uncountable chain. This implies that $R^{+}$ is not Borel linearizable, and, by Theorem 10, $R_{2} \preceq_{\mathrm{B}} R^{+}$. So if $R^{+} \preceq_{\mathrm{B}} R$, we get $R_{2} \preceq_{\mathrm{B}} R$, and Corollary 9 applies, as wanted.

So it remains to construct the sequence $\left(f_{\xi}\right)_{\xi<\omega_{1}}$ as above. Consider the subset $A$ of $\omega^{\omega}$ consisting of those functions $f$ satisfying $f(n) \leq n$ for all $n$, and $n-f(n) \rightarrow \infty$ with $n$. It is clearly enough to prove that for any sequence $\left(g_{k}\right)_{k \in \omega}$ in $A$, there is $f$ in $A$ with $\forall k \forall^{*} n g_{k}(n)<f(n)$, as one can then by using it build the $\omega_{1}$ sequence in $A$ by induction on the countable ordinals. As $A$ is closed under finite pointwise suprema, we may assume the sequence $g_{k}$ is increasing. Let then $n_{k}$ be least with $n-g_{k}(n)>k$ for all $n>n_{k}$, and let $f(n)$ be 0 for $n<n_{0}$, and $g_{k}(n)+1$ for $n_{k} \leq n<n_{k+1}$. One easily checks that $f$ works.

Remark. Corollary 11 is indeed optimal, for if $R$ is such that all $R$ chains have size $\leq k<\omega$, then $R^{+} \preceq_{\mathrm{B}} R$. To see this, note first that if $R$ has this property, then so does $R^{+}$. Moreover, it is not hard to show by induction on $k$ that if $R$ on $X$ is a $\boldsymbol{\Sigma}_{1}^{1}$ strict order with no $k+1$-chain, then $R \preceq_{\mathrm{B}}(k,<)$. This is clear if $k=1$. For $k+1$, consider the $\boldsymbol{\Sigma}_{1}^{1}$ set $A \subseteq X$ of all points which are the maximum element of a $k+1$-chain in $R$. By the hypothesis, $A$ is a subset of the $\Pi_{1}^{1}$ set $C$ of all $R$-maximal points. By separation, there is a Borel set $B$ with $A \subseteq B \subseteq C$. On the complement of $B$, there are no $k+1$-chains in $R$, so there is by induction a Borel homomorphism into $(k,<)$. Sending the points of $B$ to $k$ then gives the desired Borel homomorphism.

Finally, if $k$ is the least upper bound to the cardinality of $R$-chains, we deduce by the preceding facts that $R^{+} \preceq_{\mathrm{B}}(k,<) \preceq_{\mathrm{B}} R$, as desired.

In some cases, one can even have $R^{+} \leq_{\mathrm{B}} R$, for example if $X=\{0,1,2\}$ with $0 R 1$, as witnessed by sending the sequences which are eventually $i$, for $i=0,1$, to $i$, and the other sequences to 2 .

We now briefly indicate how to adapt the previous arguments to get the following result, which subsumes both Corollaries 9 and 11 and is valid for arbitrary $\boldsymbol{\Sigma}_{1}^{1}$ oriented graphs: 
Theorem 12. Let $R$ be a $\boldsymbol{\Sigma}_{1}^{1}$ oriented graph on a Polish space $X$. Assume that $(*)$ for all $k \in \omega$, there is a sequence $\left(x_{i}^{k}\right)_{i \leq k}$ in $X$ with $x_{i}^{k} R x_{i+1}^{k}$ for all $i<k$. Then $R^{+} k_{\mathrm{B}} R$.

Proof. We first introduce an oriented graph $G_{2}$-which replaces $R_{2}$. It is defined on the space $X_{2}^{\prime}$ of all infinite co-infinite subsets of $\omega$ by

$$
A G_{2} B \leftrightarrow A \triangle B \text { is finite \& } \operatorname{card}(B-A)=\operatorname{card}(A-B)+1 \text {. }
$$

We can then define inductively (as we did for the $R_{\xi}$ 's) graphs $G_{\xi}$, for successor $\xi \geq 2$, by setting for successor $\xi, G_{\xi+1}=G_{\xi}^{+}$, and for limit $\lambda$, with $\left(\lambda_{n}\right)$ an increasing sequence of successor ordinals converging to it, $G_{\lambda+1}=$ $\left(G_{\lambda_{n}}\right)_{n}^{+}$. We can of course view $G_{2}$ as defined on $X_{2}=2^{\omega}$, and hence $G_{\xi}$ as defined on $X_{\xi}$.

We now argue as in Corollary 11. First we check that if $R$ is a $\boldsymbol{\Sigma}_{1}^{1}$ oriented graph which satisfies condition $(*)$, then $G_{2} \preceq_{\mathrm{B}} R^{+}$. To see this, let $\left(x_{i}^{k}\right)_{i<k}$ be a witness for $(*)$, and define, for $A$ an infinite co-infinite subset of $\omega, i_{A}(k)=\operatorname{card}(A \cap k)$ and $f(A)=\left(x_{i_{A}(k)}^{k}\right)_{k \in \omega}$. Then if $A G_{2} B$ and $n=\sup (A \triangle B)+1$, one sees for $k \geq n$ that $i_{\mathrm{B}}(k)=i_{A}(k)+1$, hence $x_{i_{A}(k)}^{k} R x_{i_{\mathrm{B}}(k)}^{k}$. So $f(A) R^{+} f(B)$, and $f$ is a Borel homomorphism from $G_{2}$ to $R^{+}$.

The second step of the proof is then immediate, by induction on $\xi$ : If $R$ satisfies $(*)$ and $R^{+} \preceq_{\mathrm{B}} R$, then for all $\xi, G_{\xi} \preceq_{\mathrm{B}} R$.

So it remains to show that this is impossible, by proving that for all successor $\xi \geq 2$, ind $\left(G_{\xi}\right)>\xi$. The proof of this last fact is entirely analogous to the proof we gave for $R_{\xi}$. We just have to prove the statements analogous to Proposition 6 and Lemma 7. The analog of Lemma 7 is obvious, with the same proof, using the particular form of $G_{\xi}$. For the analog of Proposition 6, it comes down to proving that there is a continuous map $f: 2^{\omega} \rightarrow 2^{\omega} \times 2^{\omega}$ sending $L_{0}$ to $G_{2}$ and $L_{1}$ to $\check{G}_{2}$. To do this, one can use the determinacy of the following usual separation game: players I and II play $\alpha \in 2^{\omega}$ and $(\beta, \gamma) \in 2^{\omega} \times 2^{\omega}$ respectively (bit by bit), and player II wins if $\alpha \in L_{0}$ implies $\beta G_{2} \gamma$, and $\alpha \in L_{1}$ implies $\gamma G_{2} \beta$. This game is clearly Borel, hence determined, and a winning strategy for player II provides the wanted map $f$. So it is enough to check that player I does not have a winning strategy. But otherwise, we get a continuous map $g: 2^{\omega} \times 2^{\omega} \rightarrow 2^{\omega}$ which satisfies $g\left(2^{\omega} \times 2^{\omega}\right) \subseteq L_{0} \cup L_{1}, G_{2} \subseteq g^{-1}\left(L_{1}\right)=g^{-1}\left(2^{\omega}-L_{0}\right)$, and $\check{G}_{2} \subseteq g^{-1}\left(L_{0}\right)=g^{-1}\left(2^{\omega}-L_{1}\right)$, by the definition of the game. Now clearly $G_{2}$ and $\check{G}_{2}$ are both dense in $\left(X_{2}^{\prime}\right)^{2}$, so $g^{-1}\left(L_{0}\right)$ and $g^{-1}\left(L_{1}\right)$ are disjoint dense $G_{\delta}$ in it, a clear contradiction. Putting everything together, this proves Theorem 12.

To end up this paper, let us come back to our original motivation, i.e. the unboundedness property of $\boldsymbol{\Sigma}_{1}^{1}$ partial orders under $\leq_{\mathrm{B}}$ (Theorem 1), 
and discuss how this result can be generalized to other types of quasi-orders besides partial orders.

If $R$ is a $\Sigma_{1}^{1}$ quasi-order on a Polish space $X$, denote by $\equiv_{R}$ the associated (necessarily $\boldsymbol{\Sigma}_{1}^{1}$ ) equivalence relation, defined by $\equiv_{R}=R \cap \breve{R}$. And if $E$ is a given $\boldsymbol{\Sigma}_{1}^{1}$ equivalence relation on $X$, let $\mathcal{C}_{E}$ be the class of $\boldsymbol{\Sigma}_{1}^{1}$ quasi-orders $R$ on $X$ with $\equiv_{R}=E$. So partial orders correspond to the case of equality, on say $X=2^{\omega}$. And Theorem 1 says that when $E$ is equality, $\mathcal{C}_{E}$ admits no complete element. What is the situation for other E's?

First, it is proved in Louveau-Rosendal [LR] that if $E$ is a complete $\boldsymbol{\Sigma}_{1}^{1}$ equivalence relation, one has $E=\equiv_{R}$ for some complete $\boldsymbol{\Sigma}_{1}^{1}$ quasi-order $R$, which is a fortiori complete in $\mathcal{C}_{E}$. So this gives an example of a $\boldsymbol{\Sigma}_{1}^{1}$ equivalence relation $E$ for which there exists a complete element in $\mathcal{C}_{E}$. Also, the same is true at the other extreme, if $E$ has only countably many classes, for then $\mathcal{C}_{E}$ corresponds (up to Borel bi-reducibility) to countable partial orders, and it is well known that there exists a complete countable partial order.

Here we have:

Corollary 13. Let $E$ be a Borel equivalence relation on some Polish space $X$, with uncountably many classes. Then $\mathcal{C}_{E}$ has no complete element, and in fact the Borel elements in $\mathcal{C}_{E}$ are unbounded in $\mathcal{C}_{E}$.

Proof. Suppose, towards a contradiction, that some $R \in \mathcal{C}_{E}$ Borel reduces all Borel elements in $\mathcal{C}_{E}$. Consider the strict order $<_{R}=R-E$, which is $\boldsymbol{\Sigma}_{1}^{1}$ as $E$ is Borel. We get the desired contradiction by proving that $<_{R}$ Borel reduces all Borel strict orders on $2^{\omega}$, contradicting Theorem 3 . So let $S$ be a Borel strict order on $2^{\omega}$. By our assumption and Silver's theorem, there is a one-to-one continuous map $f: 2^{\omega} \rightarrow X$ which reduces equality to $E$. Define then $S^{\prime}$ on $X$ by

$$
x S^{\prime} y \leftrightarrow x E y \text { or } \exists \alpha \in 2^{\omega} \exists \beta \in 2^{\omega}(f(\alpha) E x \text { and } f(\beta) E y \text { and } \alpha S \beta) .
$$

It is easy to check that $S^{\prime}$ is a quasi-order with $\equiv_{S^{\prime}}=E$, and that $S^{\prime}$ is Borel (for the $\alpha, \beta$ in the definition are unique, when they exist). Moreover, $f$ is a witness that $S \leq_{\mathrm{B}} S^{\prime}$, and as $S^{\prime} \in \mathcal{C}_{E}$, also $S^{\prime} \leq_{\mathrm{B}} R$, hence we get the desired contradiction.

We do not know whether there is a complete element in $\mathcal{C}_{E}$ when $E$ is a $\Sigma_{1}^{1}$ equivalence relation which is neither complete nor Borel.

\section{References}

[FS] H. Friedman and L. Stanley, A Borel reducibility theory for classes of countable structures, J. Symbolic Logic 54 (1989), 894-914. 
[HMS] L. Harrington, D. Marker and S. Shelah, Borel orderings, Trans. Amer. Math. Soc. 310 (1988), 293-302

[HKL] G. Hjorth, A. S. Kechris and A. Louveau, Borel equivalence relations induced by actions of the symmetric group, Ann. Pure Appl. Logic 92 (1998), 63-112.

[K] V. Kanovei, When a partial Borel order is linearizable, Fund. Math. 155 (1998), 301-309.

[L1] A. Louveau, On the reducibility order between Borel equivalence relations, Stud. Logic Found. Math. 134 (1994), 151-155.

[L2] - Closed orders and their vicinity, in preparation.

[L3] - Some dichotomy results for analytic graphs, in preparation.

[LR] A. Louveau and C. Rosendal, Complete analytic equivalence relations, Trans. Amer. Math. Soc. 357 (2005), 4839-4866.

Institut de Mathématiques de Jussieu

Analyse Fonctionnelle, boîte 186

Université Paris 6

4 Place Jussieu

F-75252 Paris Cedex 05, France

E-mail: louveau@ccr.jussieu.fr

Received 2 June 2005;

in revised form 4 July 2006 